\title{
Structural identification of percolation of nanoparticles
}

\author{
Dafne Musino $^{1}$, Anne-Caroline Genix ${ }^{1 *}$, Edouard Chauveau, ${ }^{1}$ Thomas Bizien ${ }^{2}$, Julian Oberdisse ${ }^{1}$ \\ ${ }^{1}$ Laboratoire Charles Coulomb (L2C), Université de Montpellier, CNRS, F-34095 Montpellier, \\ France \\ ${ }^{2}$ SOLEIL Synchrotron, L'Orme des Merisiers, Gif-Sur-Yvette, 91192 Saint-Aubin, France
}

*Author for correspondence: anne-caroline.genix@umontpellier.fr

\begin{abstract}
We propose a method relying on structural measurements by small-angle scattering to quantitatively follow aggregation of nanoparticles (NPs) in concentrated colloidal assemblies or suspensions up to percolation, regardless of complex structure factors arising due to interactions. As experimental model system, the dispersion of silica NPs in a styrene-butadiene matrix has been analyzed by small-angle Xray scattering and transmission electron microscopy (TEM), as a function of particle concentration. A reverse Monte Carlo analysis applied to the NP scattering compared favorably with TEM. By combining it with an aggregate recognition algorithm, series of representative real space structures and aggregation number distribution functions have been determined up to high concentrations, taking into account particle polydispersity. Our analysis demonstrates that the formation of large percolating aggregates on the scale of the simulation box (of linear dimension $1 / \mathrm{q}_{\min }$, here micron-sized) can be mapped onto the macroscopic percolation characterized by rheology. Our method is thus capable of determining aggregate structure in dense NP systems with strong - possibly unknown - interactions visible in scattering. It is hoped to be useful in many other colloidal systems, beyond the case of polymer nanocomposites exemplarily studied here.
\end{abstract}




\section{Introduction}

Characterizing the dispersion state of nanoparticles (NPs) in suspension or in bulk - typically polymer matrices - is particularly difficult at high particle concentrations, where direct imaging methods including tomography ${ }^{1,2}$ are hampered by overlapping objects, whereas scattering methods and in particular small-angle scattering have to take into account strong and usually unknown interparticle interactions. ${ }^{3-5}$ Such interactions may lead to complex multi-scale structures consisting of NPs at the smallest length scale, which may aggregate due to attractive interactions. ${ }^{6}$ Such aggregates may grow into infinite, possibly phase separating clusters, or induce gelling of the sample. In some cases, finitesized aggregates are kinetically trapped by an increase of the matrix viscosity, ${ }^{78}$ or stabilized by longrange repulsive interactions ${ }^{9}$. Their scattering can then be described by an aggregate form factor taking into account both interparticle contact and total aggregate size, mass, density, and shape. ${ }^{10,11}$ Separated aggregates can also be visualized by electron microscopy. ${ }^{12,13}$ Aggregates may interact repulsively, ${ }^{14}$ or be assembled into agglomerates, branches, or sheets ${ }^{15}$ on an even larger scale, a striking example being the multi-scale structure in polymer nanocomposites. ${ }^{16,17}$ A particular state of matter is reached when the NPs form percolating paths across the entire sample, forming a bicontinuous structure of the (usually hard) particle phase and the matrix, which may be a solvent in case of gels, an elastomer, or any viscoelastic polymer used above its $\mathrm{T}_{\mathrm{g}}$.

Percolation has attracted strong interest of statistical physics, for various particle shapes, interactions, and dimensions of space. Simulations are either performed on lattices, ${ }^{18,} 19$ or in continuum ${ }^{20-24}$ models. In the latter case, the question of the detection of particle contact arises. It can be dealt with either by generating a priori contacting dense random arrangement of spheres, i.e., creating a supporting random "lattice" structure and studying lattice percolation as on regular lattices, for monodisperse ${ }^{25}$ or polydisperse ${ }^{21}$ spheres. Alternatively, calculations based on the Ornstein-Zernicke integral equation have triggered analytical approaches for different interparticle interactions. ${ }^{26,27}$ In these theories, connections between spheres are identified by overlap between either permeable or non-interacting spheres. Alternatively, connectivity can be defined by some maximum surface-tosurface distance as discussed for rod-like systems ${ }^{28}$ or hard spheres ${ }^{29}$ including a smooth transition representing tunneling in electric conductivity. ${ }^{30}$ In simulations this distance is regarded as a free parameter, which could be fixed by experimental evidence as intended here. These approaches predict a percolation threshold $\Phi_{c}$, i.e., a critical volume fraction of NPs (fraction of occupied space) above which percolating paths exist, as well as details on the power law approach of percolation. ${ }^{20,31}$ For a random packing of hard spheres, a threshold of ca. $18 \%$ in volume fraction was found. ${ }^{25}$ This value can be compared to lattice models mapped onto monodisperse spheres, which predict thresholds of ca. $15 \% \mathrm{v}$ for the common 3D lattices. ${ }^{19}$ Sphere polydispersity (in 3D) induces a weak decrease of the threshold. ${ }^{21,32}$ Experimentally, percolation is most conveniently studied by transport properties, i.e. dynamics, like in particular conductivity, or more generally dielectric spectroscopy. ${ }^{33,34}$ Another 
method is rheology, ${ }^{35}$ where force transmission (i.e., transport of momentum) allows detecting percolation by the formation of hard NP paths stiffening the sample, like e.g. in filled elastomers or entangled polymers, ${ }^{16,36}$ with direct impact on the viscoelastic response. The analysis of static structure is usually not used for this purpose, because the structure of the entire macroscopic sample can usually not be characterized, and because the structure of a percolating path is usually not much different from one which is interrupted. Moreover, percolation as a statistical phenomenon is difficult to apprehend in a single structural snapshot. It is our intention to show in this article that small-angle neutron or X-ray scattering allows the identification of percolation in concentrated nanoparticle suspensions. This seems a priori counter-intuitive, due to the lack of detail on local particle positions obtained by such low resolution methods. Its major advantage, however, lies in the averaging of the microstructure over macroscopic sample volumes present in the beam, allowing to probe the statistical nature of percolation.

As an exemplary system for particle aggregation and percolation, the dispersion of hard nanoparticles in polymer matrices is studied in this article. Because of scattering being sensitive to particles regardless of their environment (contrast), this can be generalized to suspensions in any medium. Particle structure in polymer results from a combination of the starting conditions - well-dispersed model NPs ${ }^{7,37}$ or aggregated powders ${ }^{16,38}$-, the input of mechanical energy in a mixing process to separate and distribute NPs, and the physical chemistry of the particle-polymer interface affecting particle-particle and particle-polymer interactions. ${ }^{39,40}$ The NP surface can be modified chemically, in particular via the grafting of either small, usually surface-active molecules also called coating agents, ${ }^{41}$ or long polymer chains. ${ }^{15,42}$ Such surface modifications influence the final NP dispersion by changing the electrostatic charge of the particles by consumption of surface OH-groups, ${ }^{43}$ by modifying their van der Waals interactions, ${ }^{44}$ or introducing steric repulsion. ${ }^{15}$ Surface modifications have attracted considerable interest in the formulation of polymer nanocomposites (PNCs), ${ }^{45}$ in order to control NP dispersion and thus mechanical properties ${ }^{46,47}$ via aggregation and/or percolation, ${ }^{36,38}$ or optical properties related to structural heterogeneities on the scale of NP aggregates. ${ }^{48}$ Note here that the presence of an interphase between NPs and bulk polymer is a key factor affecting the macroscopic properties in PNCs, both in terms of structure and dynamics. ${ }^{49,50}$

We have shown recently that the structure of simplified industrial polymer nanocomposites produced by solid-phase mixing of styrene-butadiene (SB) and silica can be tuned by means of a synergetic effect of two "small" molecules, diphenyl guanidine and triethoxy octylsilane, which are commonly used in car tires. ${ }^{51}$ Here, "simplified" refers to a limited number of ingredients with a styrenebutadiene (SB) polymer of well-defined mass generating clear rheological features, while "industrial" characterizes the disordered silica filler of industrial origin. In PNCs made by solvent casting using well-defined colloidal silica grafted with silane-molecules, the dispersion in a SB matrix was recently studied at low concentration using a combination of small-angle X-ray scattering (SAXS) and reverse 
Monte Carlo (RMC). ${ }^{52}$ In particular, the impact of functionality (mono- or tri-functionality) of silanes of identical hydrophobicity was explored. The effect of concentration on model bare nanoparticles incorporated into a SB matrix with a complete control of colloidal stability throughout the solventcasting protocol has not been addressed. Thus, percolation was out of reach.

Our original SAXS/RMC analysis of nanoparticle assemblies presented here allows the determination of average aggregation numbers of polydisperse nanoparticles even at high concentrations. We demonstrate that it is possible to identify percolation with a static structural method on the scale of $1 / q_{\min }$, in agreement with rheological measurements of the storage modulus, and supported by electron microscopy. These results are compared to the case of ideal hard spheres of same polydispersity, i.e., which respect excluded volume conditions and the contact of which is detected using the same maximum distance criterion. This comparison allows cross-checking our approach against literature simulation results on randomly dispersed spheres, and in addition highlights the effect of interactions between real NPs in the experimental nanocomposite system.

\section{Experimental and simulation}

Nanoparticle suspension. Colloidal silica NPs (Ludox ${ }^{\circledR}$ TM40 from Sigma-Aldrich) have been characterized by SAXS in de-ionized water at $1 \% \mathrm{v}\left(\mathrm{R}_{0}=12.5 \mathrm{~nm}, \log\right.$-normal polydispersity $\sigma=$ $0.12)$. The diluted suspension of bare NPs $(2.2 \% \mathrm{v}$ in water) was transferred into pure ethanol and then into MEK by successive dialysis ( $24 \mathrm{~h}$ for each step, MEK twice) and sonicated for 30 minutes at room temperature. The residual amounts of $\mathrm{H}_{2} \mathrm{O}$ and ethanol in the final dialysis suspension determined by NMR were below $2.5 \% \mathrm{v}$. The dispersion state in MEK was measured by SAXS and discussed in our earlier work; ${ }^{52}$ it will be compared with the PNC results in the next section.

Polymer and nanocomposite formulation. Highly monodisperse styrene-butadiene random copolymer was purpose-synthesized by Synthos $(177 \mathrm{~kg} / \mathrm{mol}$, polydispersity index $=1.02$, styrene units $19.1 \% \mathrm{w}$, butadiene $80.9 \% \mathrm{w}$, out of which $42.6 \% \mathrm{w}$ are 1,4 -units and the rest 1,2$)$. The polymer was dissolved in MEK (10\%v), then mixed with the NP suspension in MEK at $1 \% \mathrm{v}$, followed by solvent casting on a Teflon support for $24 \mathrm{~h}$ at $50^{\circ} \mathrm{C} .{ }^{52}$ The final PNC samples have a typical size of $3 \mathrm{~cm}$ diameter with a thickness of ca. $100 \mu \mathrm{m}$.

Structural analysis. Small-angle X-ray scattering was performed on beamline SWING at synchrotron SOLEIL (Saint Aubin, France) using standard conditions (sample-to-detector distances $2 \mathrm{~m}, 5 \mathrm{~m}$ and $6.5 \mathrm{~m}$; wavelength $1 \AA$, giving a q-range from $6.210^{-4}$ to $5.610^{-1} \AA^{-1}$ ). Standard data reduction tools given by Soleil were used (Foxtrot 3.1). Matrix contributions have been measured independently, and subtracted. For comparison of particle scattering in different solvents or polymer, at different particle 
concentrations, intensities have been normalized to the form factor scattering observed at $1 \% \mathrm{v}$ in water. The normalized intensity $I_{\text {norm }}$ then reads:

$$
\mathrm{I}_{\text {norm }}(\mathrm{q})=\mathrm{I}_{\exp }(\mathrm{q}) \frac{\Phi_{\text {form }}}{\Phi}\left(\frac{\Delta \rho_{\text {form }}}{\Delta \rho}\right)^{2}
$$

where the index 'form' corresponds to the form factor measurement, and $\Phi$ and $\Delta \rho$ to the particle volume fraction and contrast of the originally measured intensity $\mathrm{I}_{\exp }$. The scattering length densities used to calculate the contrasts $\Delta \rho=\rho_{\mathrm{SiO} 2}-\rho_{\text {medium }}$ are: $\rho_{\mathrm{SiO} 2}=18.910^{10} \mathrm{~cm}^{-2}, \rho_{\mathrm{H} 2 \mathrm{O}}=9.510^{10} \mathrm{~cm}^{-2}, \rho_{\mathrm{EtOH}}=$ $7.610^{10} \mathrm{~cm}^{-2}, \rho_{\mathrm{MEK}}=7.710^{10} \mathrm{~cm}^{-2}$, and $\rho_{\mathrm{SB}}=8.910^{10} \mathrm{~cm}^{-2}$.

Transmission electron microscopy (TEM) was performed on samples prepared by ultracryomicrotomy at $-140{ }^{\circ} \mathrm{C}$ (Leica EM UC7, Diatome Cryo 35, desired thickness set to $70 \mathrm{~nm}$ ), deposited on copper grids with carbon membrane, using a JEOL 1200 EXII apparatus at $100 \mathrm{kV}$ and a Quemesa camera (11 Mpix) from SIS Olympus.

Reverse Monte Carlo scattering analysis. Spatial distributions of polydisperse hard spheres of scattering compatible with the measured intensities have been determined using a reverse Monte Carlo 14, 53-55 simulation, and converted into distribution functions of aggregate mass by an aggregate recognition algorithm. ${ }^{52}$ Details and fine-tuning of the latter are given in the results section. The cubic box size with periodic boundary conditions was set by the experimental minimum q-value, $\mathrm{L}_{\mathrm{box}}=$ $2 \pi / \mathrm{q}_{\min }$, which in turn determines the number of particles given the experimental silica volume fraction, $\Phi$. To fix ideas, about 1200 particles correspond to $1 \% \mathrm{v}$ with the present $\mathrm{q}_{\min }$, and proportionally higher numbers for higher silica contents, up to ca. 20'000. The log-normal size distribution function was determined from the intensity measurement of a dilute and non-aggregated suspension, and it was used to generate the particles. The intensity was calculated taking into account polydispersity, i.e., correctly weighting all partial structure factors. ${ }^{11}$ The natural base unit of the calculation is thus a sphere drawn from the experimental size distribution, which allows the construction of any types of aggregate or dispersion. The initial configuration has been defined with a pre-aggregation parameter $\alpha=5 \%$ (see ${ }^{52}$ for details). This parameter has been introduced in order to accelerate the convergence of the algorithm in presence of aggregation, but does not predefine the final state. Pre-aggregation promotes aggregation around $\alpha=5 \%$ of randomly set "seed" particles. After defining such an initial configuration, the RMC procedure lets the system evolve by optimizing the particle positions within the cubic simulation box with periodic boundary conditions, until agreement between the experimental and simulated intensities is obtained within an allowed accuracy. By continuing the random particle displacements under the condition of agreement with the experimental intensity, a sequence of statistically equivalent particle configurations is obtained. These can then be averaged to obtain smooth intensities, and configurational averages. For comparison, random dispersions of particles (of same size and polydispersity) only obeying the excluded volume 
condition - and thus not related to the experimental intensities - have been generated and analyzed using the same aggregate recognition algorithm.

Rheology. The rheological response of the nanocomposites was obtained with a stress-controlled rheometer AR 2000 used in the strain-controlled mode. We used a plate-plate geometry with $20 \mathrm{~mm}$ diameter of stainless steel. Isothermal frequency sweeps from 100 to $0.1 \mathrm{rad} / \mathrm{s}$ at fixed low deformation level in the linear regime (below 1\%) were performed in the temperature range from 10 to $80{ }^{\circ} \mathrm{C}$ with an accuracy of $\pm 0.1 \mathrm{~K}$. Using the principle of time-temperature superposition, master curves of the storage modulus, $\mathrm{G}^{\prime}(\omega)$, and the loss modulus, $\mathrm{G}^{\prime \prime}(\omega)$, corresponding to measurements at $50{ }^{\circ} \mathrm{C}$ were established.

\section{Results and discussion}

Rheology in shear geometry is a method capable of probing large-scale percolation by detecting hardening via increase of the storage modulus. The evolution with $\Phi$ of the shear moduli, G' and G' $(\omega)$, of the silica-SB PNCs is shown in Figure 1a for selected silica volume fractions at the reference temperature of $50^{\circ} \mathrm{C}$. As usually observed, ${ }^{56,57}$ the addition of filler NPs increases the lowstrain storage modulus. This is the so-called reinforcement effect that is accompanied by a fluid to solid-like transition in the terminal regime, ${ }^{58}$ as well as by a high $G^{\prime}$ with respect to the viscous component G' with increasing silica content. From the high-frequency storage modulus $\mathrm{G}_{0}{ }^{\prime}-$ estimated here at the frequency value where $G^{\prime \prime}(\omega)$ displays a minimum -, the relative reinforcement of the nanocomposites $\mathrm{G}_{0}{ }^{\prime} / \mathrm{G}^{\prime}{ }_{0}$ matrix with respect to the pure $\mathrm{SB}$ matrix has been calculated as a function of silica volume fraction. These reinforcement factors are given in Figure 1b. At low $\Phi$, they lie above the Einstein-prediction adapted by Smallwood of $1+2.5 \Phi,{ }^{59}$ indicating that $\Phi$ of the reinforcing hard material is higher than the nominal one. Including the polymer inside aggregates by replacing the Einstein-expression by $1+2.5 \Phi / \kappa$ gives a correct description with $\kappa=25 \%$ as shown in Figure $1 \mathrm{~b}$. At higher $\Phi$, the strong increase above the modified Einstein-expression suggests higherorder interactions between NPs in the polymer matrix, and in particular large-scale structuring in aggregates or networks. The system evolves towards percolation, and the percolation threshold is commonly identified ${ }^{56}$ with the strong deviation from hydrodynamic reinforcement as indicated by the dashed line in Figure $1 \mathrm{~b}$. The resulting rheological percolation threshold is $\Phi_{\mathrm{c}}^{\text {rheo }}=12.5 \% \mathrm{v}$, which is the value used in the analysis of the SAXS data latter in this article to define NP contact. 

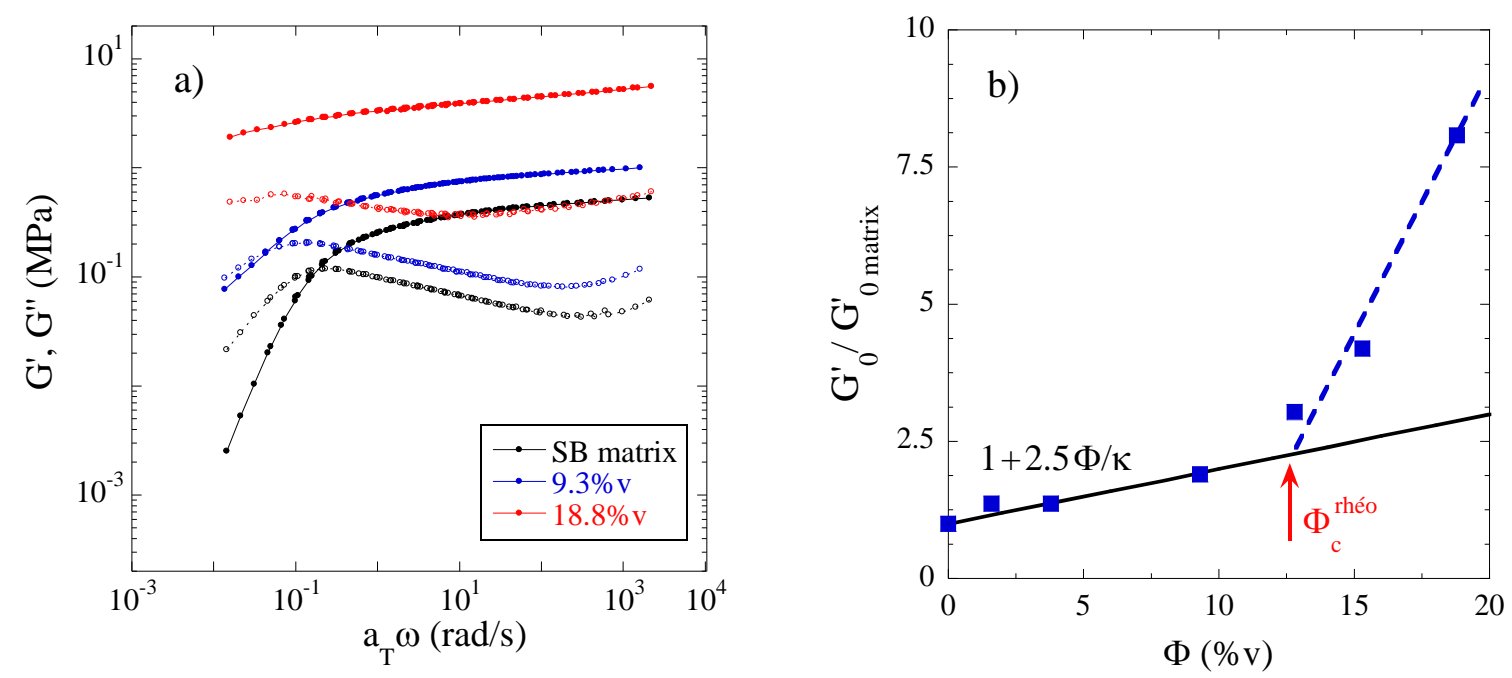

Fig. 1 a) G' (plain) and G" (empty symbols) as a function of $\omega$ at the reference temperature of $50^{\circ} \mathrm{C}$, for the SB matrix and two PNCs as indicated in the legend. b) Rheological reinforcement factor of silica-SB nanocomposites as a function of NP volume fraction. The plateau modulus has been taken at the position of the G" minimum at the reference temperature of $50^{\circ} \mathrm{C}$. The solid line is the Einstein prediction with $\kappa=25 \%$, and the dashed line illustrates the strong increase above $\Phi_{c}^{\text {rheo }}$.

As the composition of the samples studied in Figure $1 \mathrm{~b}$ is unchanged (same silica, same polymer), the structure of these samples must vary as the silica content is increased, and the quantitative analysis of the structure on the microscale is the main purpose of this article. Although it does not provide statistical averages like SAXS, TEM gives an idea of the typical aggregation state of the particles. In Figure 2, TEM pictures of PNCs of typical slice thickness $70 \mathrm{~nm}$ are shown for different silica fractions ranging from $2 \% \mathrm{v}$ to about $20 \% \mathrm{v}$. For comparison, simulation results discussed below based on experimental SAXS are compared to each TEM picture at the same NP volume fraction. These pictures illustrate that particle configurations obtained by SAXS and RMC are representative of the real structures. While the NPs in Figure 2a are mostly individual, or form small isolated aggregates, connecting paths may exist at intermediate concentrations (Figures $2 \mathrm{~b}$ and in particular $2 \mathrm{c}$ ), and certainly the crowded silica NPs at $20 \%$ (Figure 2d) are completely connected. It is concluded that 3D percolation takes place between $10 \%$ and $20 \%$ NP volume fraction. However, the influence of $\Phi$ is difficult to evaluate by TEM, due to the lack of statistics and the observation of thin slices of sample may shift conclusions - in this extent scattering is more trustworthy. Therefore, we will now perform the concentration study by SAXS. 


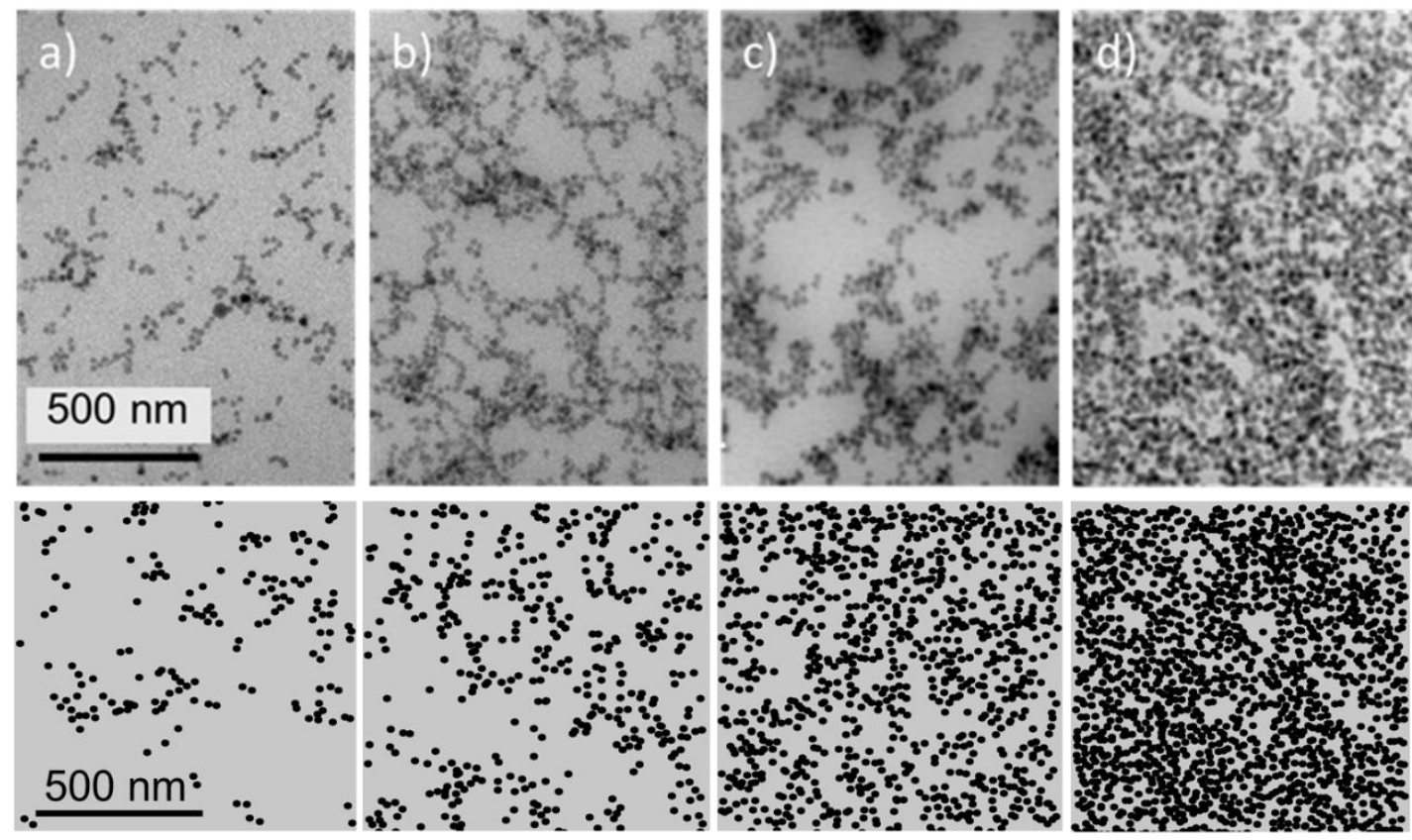

Fig. 2 TEM pictures of silica-SB nanocomposites with increasing volume fraction. (a) $2.6 \% \mathrm{v}$, (b) $6.0 \% \mathrm{v}$, (c) $10 \% \mathrm{v}$ and (d) $20 \% \mathrm{v}$. For illustration, $70 \mathrm{~nm}$-thick slices of the RMC-simulation box are shown below each picture, at the same nominal concentrations.

The scattered intensities for PNC samples with different values of $\Phi$ up to $20 \% \mathrm{v}$ are shown in Figure 3a. The scattered intensities evolve as follow: the higher $\Phi$, the lower the low-q intensity, the lower the slope at intermediate q, and the deeper the dip around $0.016 \AA^{-1}$. This dip is termed the "correlation hole", and its increasing depth indicates locally higher NP concentrations. ${ }^{11}$ With increasing $\Phi$, the shape of the curves remains similar, i.e. there are no clear repulsive interactions peaks, and the low-q shoulder stays approximately in the same q-range. The decrease in the low-q structure (below $2.10^{-3} \AA^{-}$ $\left.{ }^{1}\right)$ is due to the repulsive interactions between NP aggregates, which dominate at higher concentrations. 

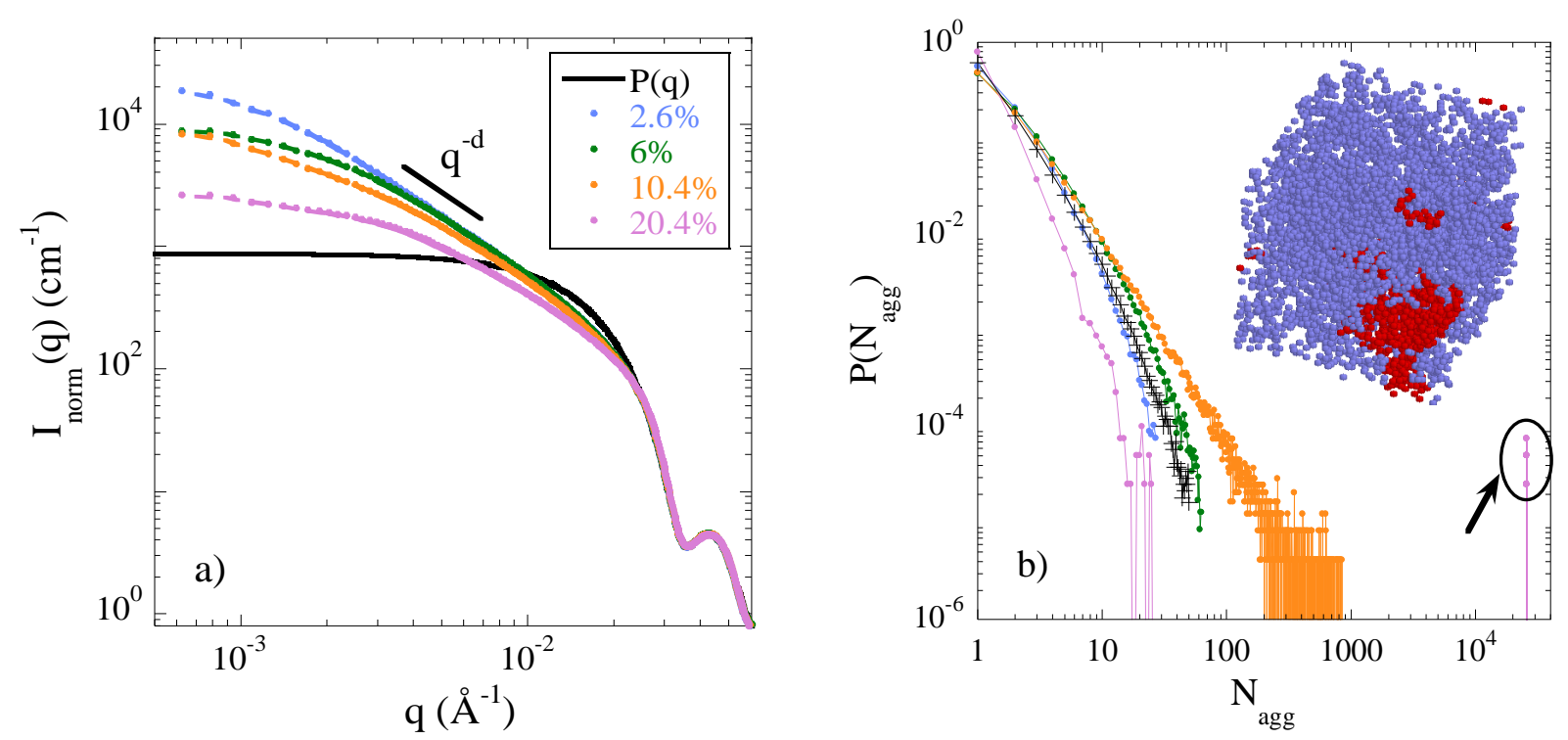

Fig. 3 (a) SAXS scattered intensities normed to NP form factor in water (black line) of silica-SB nanocomposites, for different NP volume fractions as indicated in the legend. The dotted lines superimposed to the symbols represent the result of the RMC fit. (b) Distribution functions of $\mathrm{N}_{\text {agg }}$ deduced from scattering $(\delta=$ $0.6)$, normalized by the total number of aggregates in each configuration, and averaged over 100 configurations. The distribution for a random dispersion of the same NPs $(\Phi=10.4 \% \mathrm{v}, \delta=0.6)$ is represented by black crosses. Inset: snapshot of the simulation box with identification of one aggregate in red. The arrow indicates completely percolated states.

The quantitative analysis of the scattering curves shown in Figure $3 \mathrm{a}$ is not an easy task, as the underlying configurations in direct space may present a distribution of polydisperse and interacting aggregates. Moreover, interactions between aggregates may depend on concentration, generating unknown structure factor contributions in the low-q intensity. It is thus necessary to discuss these data with a more precise tool, based on an inversion of the intensities into real-space configurations, and this will be performed next.

The scattering functions plotted in Figure 3a can be inverted stochastically into representative realspace assemblies of NPs populating the simulation box as defined in the Methods section. At each step of the calculation, the scattering function is calculated taking into account all the partial structure factors between spheres of different radius due to polydispersity. Once the algorithm converges, its result is a stochastic sequence of particle configurations in the simulation box compatible with the scattering, as one can judge from the dotted lines perfectly superimposed to all data sets in Figure $3 \mathrm{a}$. The number of particles in the box is fixed by the minimum q-value and $\Phi$. Therefore, statistical significance of the simulation data is obtained by letting the system evolve and average over configurations in time, under the boundary condition of agreement with the scattering. Naturally, the amount of information contained in the scattering function is less than the number of coordinates of particles in the box, but by averaging over many equivalent configurations, statistically robust features of the NP assemblies like the aggregate distribution functions defined below can be extracted. 
A first simple analysis of the RMC results is to look at the spatial distribution of particles in the simulation box - which however is far too crowded to allow for any conclusion, see the snapshot of a box in the inset of Figure 3b, where an aggregate is highlighted for illustration. As in the experiment, thin slices may be observed. This approach is illustrated by the bottom panels of Figure 2. The slices have the lateral size of the simulation box, and the same nominal thickness as the experimental samples studied by TEM. We have checked that they are statistically representative by comparing many different slices, and that the average number of NPs in each slice corresponds to the particle volume fraction. Note that the particle polydispersity is respected in the calculation but not in the graphical representation. Nonetheless, the capacity of small-angle scattering to grasp the distribution of matter in space is remarkably well illustrated by the slices resulting from the RMC-SAXS analysis shown in Figure 2: at the lowest concentration, some isolated aggregates are found, which begin to connect in the $6 \%$-sample. At $10 \%$, they seem to connect across the entire field of vision, with some voids appearing. At the highest concentration, finally, the sample is densely packed with silica NPs, while the presence of voids persists both in the TEM image and in the simulated slice deduced from the SAXS analysis by RMC.

A possible quantitative analysis of the series of particle configurations produced by the RMCsimulation are the aggregation distribution functions $\mathrm{P}\left(\mathrm{N}_{\mathrm{agg}}\right)$ shown in Figure $3 \mathrm{~b}$. These functions give the average number fraction an aggregate of given aggregation number $\mathrm{N}_{\mathrm{agg}}$ is found in the simulation box (box size ca. $1 \mu \mathrm{m}$ ), including isolated NPs with $\mathrm{N}_{\mathrm{agg}}=1$. Averages are obtained using an aggregate recognition algorithm from the series of particle configurations in the course of the simulation. The moments of this distribution functions will be convenient indicators in the following discussion. The first moment is the average aggregation number, $\left\langle\mathrm{N}_{\mathrm{agg}}\right\rangle$; the second moment, $\left\langle\mathrm{N}_{\mathrm{agg}}{ }^{2}\right\rangle$, will serve to express the width of the distribution. The ratio of these moments is sensitive to the bigger aggregates formed at high $\Phi$, and it also corresponds to the scattered intensity prefactor in absence of interactions between aggregates.

Before quantifying the state of aggregation of a particle configuration using the aggregate recognition algorithm, one needs to define a critical surface-to-surface distance $\delta\langle\mathrm{R}\rangle$ (where $\langle\mathrm{R}\rangle$ is the average NP radius) below which particles are considered as aggregated, as discussed by Otten et al. ${ }^{28}$ It is tempting to set this distance to zero, but due to the finite resolution of the scattering and particle polydispersity, non-contacting particles in close vicinity lead to a similar scattering signature, i.e., one cannot discriminate 'real contact' from 'close-to-contact'. Moreover, looking at the TEM pictures in Figure 2, it appears that some NP assemblies are likely to be counted as aggregates in scattering, in the sense that they possess centers-of-mass with a high degree of correlation, forming a dense zone. It is difficult, however, to decide from TEM if these NPs really touch, and some finite distance $\delta<\mathrm{R}\rangle$ is needed to define a contact criterion. The average particle radius seemed to be an appropriate choice of the order of magnitude of the critical surface-to-surface distance, and $\delta=1$ has been chosen in our first 
approach. ${ }^{52}$ This was a compromise motivated by the fact that critical distances too close to zero fail to detect any aggregation, whereas taking the diameter as contact parameter results in counting all NPs as one aggregate already at low $\Phi$, and the optimum value probably lies close to $\langle\mathrm{R}\rangle$.

In the present work, we have varied $\delta$ systematically between 0.4 and 2, and determined the average aggregation expressed by the ratio $\left\langle\mathrm{N}_{\mathrm{agg}}{ }^{2}\right\rangle \mid\left\langle\mathrm{N}_{\mathrm{agg}}\right\rangle$ as a function of $\Phi$. The results are shown in Figure 4 , determined for each experimental scattered intensity at various $\Phi$. Not surprisingly, the larger the $\delta$, the earlier the increase in aggregation, up to percolation on the scale of the simulation box. Percolation on this scale is identified as the formation of one huge aggregate of the size of the simulation box, i.e., the indicator $\left\langle\mathrm{N}_{\mathrm{agg}}{ }^{2}\right\rangle \mid\left\langle\mathrm{N}_{\mathrm{agg}}\right\rangle$ saturates close to the total number of particles in the box, which is $\Phi$ dependent (typically $2 \times 10^{4} \mathrm{NPs}$ at $20 \% \mathrm{v}$ ), as illustrated in Figure $3 \mathrm{~b}$ for the highest $\mathrm{N}_{\text {agg. }}$. We have therefore investigated the threshold above which clusters capable of spanning the entire box volume may exist. Such an aggregate has thus a linear dimension of $\mathrm{L}_{\mathrm{box}}$, and using a simple fractal model of spheres this can be related to a typical aggregation number:

$$
\mathrm{N}_{\mathrm{agg}}=\left(\frac{\mathrm{R}_{\mathrm{agg}}}{<\mathrm{R}>}\right)^{\mathrm{d}}
$$

The special case $\mathrm{R}_{\mathrm{agg}}=\mathrm{L}_{\mathrm{box}} / 2$ and using a fractal dimension d between 1.8 and 2.1 in order to cover limiting regimes ${ }^{60}$ of diffusion or reaction limited aggregation yields a critical aggregate mass of ca. 1000 particles. This corresponds thus to a significant fraction of the particles in the box, typically one tenth for thresholds close to $10 \%$. Note that a fractal dimension of 1.6 is found from the low concentration SAXS data in Figure 3a. In Figure 4, we have thus drawn a horizontal limiting line at $\left\langle\mathrm{N}_{\mathrm{agg}}{ }^{2}\right\rangle \mid\left\langle\mathrm{N}_{\mathrm{agg}}\right\rangle=1000$, expressing the onset of percolation on this scale. Given the steepness of the functions shown in semi-log scale on Figure 4, the exact position of the horizontal limiting line (and thus of the fractal dimension used for this estimation) is not of importance. By comparing to the position of the increase in modulus measured by rheology as discussed above $\left(\Phi_{\mathrm{c}}^{\text {rheo }}=12.5 \% \mathrm{v}\right)$, the value of the simulation parameter has been fixed to $\delta=0.6$. This is a more precise estimate than the previous one of $\delta=1 .{ }^{52}$ Note that $\delta$ is a simulation parameter reflecting the impossibility of SAXS to determine exact contact between NPs due to polydispersity and finite resolution. 


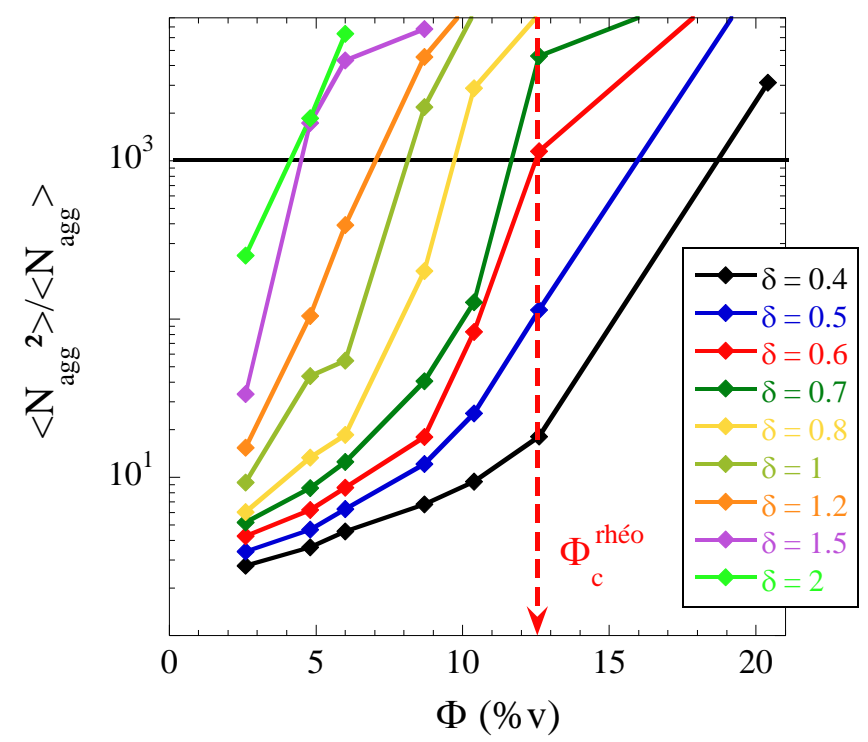

Fig. $4\left\langle\mathrm{~N}_{\mathrm{agg}}{ }^{2}\right\rangle \mid<\mathrm{N}_{\mathrm{agg}}>$ determined from aggregation number distribution functions $\mathrm{P}\left(\mathrm{N}_{\mathrm{agg}}\right)$ obtained by RMC analysis of experimental SAXS intensities, for different simulation parameters $\delta$ as indicated in the legend. The horizontal line shows the onset of percolation.

The distributions of aggregation numbers $\mathrm{P}\left(\mathrm{N}_{\mathrm{agg}}\right)$ obtained with $\delta=0.6$ from the RMC-configurations, and averaged over 100 configurations, have been shown in Figure $3 \mathrm{~b}$ for the silica-SB nanocomposites. In this graph, they are compared to the distribution of a random dispersion at $10.4 \%$ volume fraction, where large-scale aggregates are present only by fortuitous contact between NPs as recognized using the same parameter, $\delta=0.6$. The difference between the theoretical function for random dispersions (black crosses) and the ones corresponding to the measured intensities at exactly the same concentration (orange curve in Figure 3b) thus highlights the propensity of the NPs to aggregate: $\left\langle\mathrm{N}_{\mathrm{agg}}{ }^{2}\right\rangle \mid\left\langle\mathrm{N}_{\mathrm{agg}}\right\rangle=6$ and 83 , for random hard spheres and PNCs, respectively, with a tail extending towards higher aggregation numbers for the latter.

The distribution functions in Figure $3 b$ are the real-space analogues of the intensities in Figure $3 a$ describing the mass of aggregates. They allow disregarding interactions between aggregates, i.e., the mutual aggregate positions in space. The RMC procedure thus amounts to identifying aggregate mass distribution independently of any (partial) structure factor reflecting their dispersion. Moreover, it is striking to see that percolation is identified by aggregate recognition based on (static) SAXS, whereas it is usually characterized by dynamic methods accessing transport properties, like electrical conductivity, ${ }^{61}$ dielectric processes ${ }^{33}$ or stress. ${ }^{58,62}$ This is due to the use of statistical information on occupation of space on the scale of the finite-sized regions defined by $\mathrm{q}_{\mathrm{min}}$.

The evolution of the aggregate distribution expressed by $\left\langle\mathrm{N}_{\mathrm{agg}}{ }^{2}\right\rangle /\left\langle\mathrm{N}_{\mathrm{agg}}\right\rangle$ as a function of $\Phi$ is studied in Figure 5 for $\delta=0.6$, where it is also compared to that of randomly dispersed particles with excluded volume. On log-scale, a weak increase is found at low $\Phi$. Hence, small aggregates exist in these PNCs, increasing with concentration. At intermediate $\Phi$, a strong increase over two orders of magnitude is 
observed, up to saturation indicating percolation on the scale of the simulation box. The position of this increase depends on the interactions between NPs. For the random (calculated) samples, the upturn is found at ca. $15.5 \% \mathrm{v}$, which compares favorably with the threshold of $15.2 \% \mathrm{v}$ found in the literature ${ }^{30}$ for a random dispersion of monodisperse hard spheres, thus validating our calculation based on the threshold for $\left\langle\mathrm{N}_{\mathrm{agg}}{ }^{2}\right\rangle \mid\left\langle\mathrm{N}_{\mathrm{agg}}\right\rangle$ and the contact criterion $\delta=0.6$. In the experimental PNC system, the hydrophilic silica NPs experience attractive interactions in the hydrophobic polymer matrix. These interactions trigger some aggregation and finally percolation at $12.5 \% \mathrm{v}$ detected using the same contact parameter. Percolation is thus found to set in earlier than in the case of hard spheres, due to the incorporation of polymer within aggregates, thereby increasing their effective volume fraction as already found in rheology discussed in Figure 1. Finally, a higher value of $\left\langle\mathrm{N}_{\mathrm{agg}}{ }^{2}\right\rangle \mid\left\langle\mathrm{N}_{\mathrm{agg}}\right\rangle$ indicating pre-aggregation is also found at high dilution in the MEK precursor suspension for the silica NPs (see inset of Figure 5). The state of aggregation appears thus to be generated already in suspension, and is then transferred into the polymer nanocomposite.

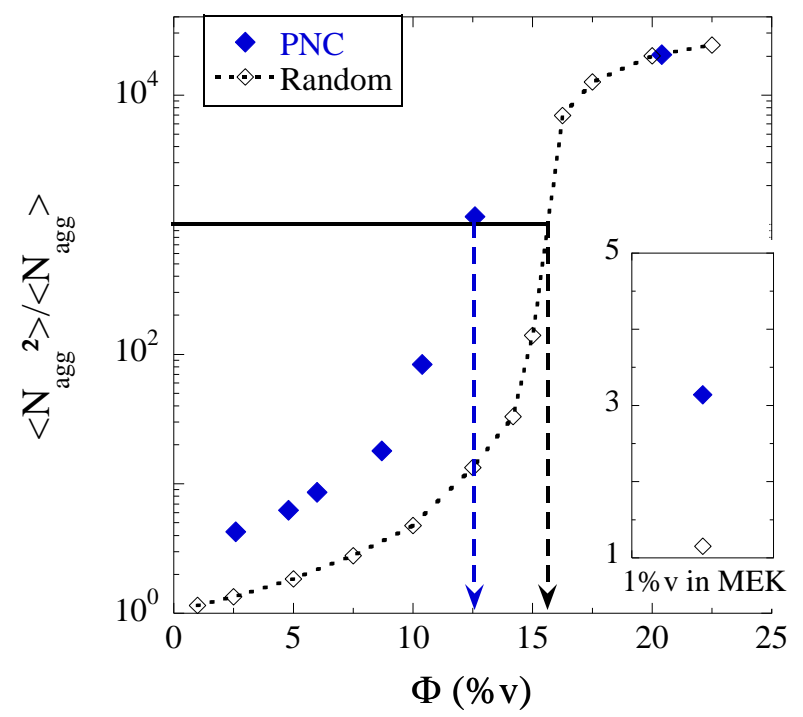

Fig. $5\left\langle\mathrm{~N}_{\mathrm{agg}}{ }^{2}\right\rangle /\left\langle\mathrm{N}_{\mathrm{agg}}\right\rangle$ as a function of silica volume fraction $\Phi$ for the silica-SB nanocomposites. The dotted line illustrates the aggregation of the same NPs with a random dispersion in the simulation box. The vertical arrows point toward $\Phi_{\mathrm{c}}$ for both sets of NPs. Inset: Same results for dilute precursor MEK suspensions.

\section{Conclusion}

We have proposed a method based on a measurement of the static structure of nanoparticle assemblies and suspensions by small-angle scattering assisted by reverse Monte Carlo computer simulations allowing the identification of percolation in agreement with macroscopic observation by rheology. The analysis is based on a parameter of particle contact $\delta<\mathrm{R}>$, i.e., the maximum surface-to-surface distance still counted as contact. As an example, the dispersion of NPs in a styrene-butadiene polymer matrix has been studied using rheology, TEM, and SAXS. TEM slices can be used to visually check 
the existence of percolating paths across the sample on the scale of approximately a micron. Moreover, the favorable comparison to equivalent slices produced from the RMC-configurations based on SAXS validates the SAXS/RMC analysis. The lack of averaging in TEM, however, makes it statistically less significant than scattering, and the transmission pictures of two dimensional slices tend to display percolation at higher concentrations. An extended image analysis of many slices taking into account the lower dimensionality of observation is largely outside the scope of this article. Nonetheless, the series of samples regrouped here does present the emergence of percolating paths: At the highest silica content of $20 \% \mathrm{v}$ the formation of a percolated network is clearly visible on the TEM picture, suggesting that percolation occurs between 10 and $20 \% \mathrm{v}$ in agreement with our analysis of static structure by SAXS.

Aggregation was characterized quantitatively. The RMC algorithm provides a 3D-structural model for the description of interacting polydisperse NPs and aggregates at any concentrations, regardless of complex interactions and resulting structure factors. The stochastic series of particle configurations reproducing the scattering were analyzed in terms of the aggregate mass distribution function, and in particular the combination of its first moments, $\left\langle\mathrm{N}_{\mathrm{agg}}{ }^{2}\right\rangle \mid\left\langle\mathrm{N}_{\mathrm{agg}}\right\rangle$. Percolation on the scale of the simulation box was identified by the formation of box-spanning aggregates as the signature of the divergence of $\left\langle\mathrm{N}_{\mathrm{agg}}{ }^{2}\right\rangle \mid\left\langle\mathrm{N}_{\mathrm{agg}}\right\rangle$. Comparison of the percolation threshold to rheological results allowed a precise determination of the NP contact criterion $\delta=0.6$. Furthermore, application of the same contact parameter to random dispersions of hard spheres gave a percolation threshold in agreement with simulations and theory for hard sphere suspensions. ${ }^{29}$ The shift in the threshold value to lower volume fractions in the real system can thus be attributed to the non-random, aggregated nature of the nanoparticles. In this case, the existence of polymer inside aggregates decreases the compacity of the latter, and thus pushes the system closer to percolation. Incidentally, we are not aware of any static technique identifying percolation, which is usually associated with transmission of stress leading to reinforcement, or transmission of current.

It is hoped that experimental progress made with reverse Monte Carlo analysis of the scattering of interacting polydisperse objects with aggregate recognition will promote a deeper understanding of NP interactions and percolation, both in suspensions and in bulk materials.

Acknowledgements. The authors are thankful for support by the ANR NANODYN project, grant ANR-14-CE22-0001-01 of the French Agence Nationale de la Recherche. We warmly thank Dimitris Vlassopoulos (Forth, Department of Materials Science and Technology, University of Crete) for help with rheology including a stay of DM, as well as critical reading of the first version of this manuscript. Access to TEM measurements at the University of Montpellier and support by F. Godiard and V. Richard are acknowledged. The polymer was a gift from Synthos, and Radoslaw Kozak, Nathalia 
Meissner, and Pawel Weda (Synthos) are thanked for polymer synthesis. Discussions with Laurent Guy and Thomas Chaussée (Solvay) are gratefully acknowledged.

\section{References}

1 H. Jinnai, Y. Shinbori, T. Kitaoka, K. Akutagawa, N. Mashita and T. Nishi, Macromolecules, 2007, 40, 6758-6764.

2 F. Dalmas, N. Genevaz, M. Roth, J. Jestin and E. Leroy, Macromolecules, 2014, 47, 2044-2051.

3 P. Lindner and T. Zemb, Neutrons, X-ray and Light Scattering, North Holland, Elsevier, Amsterdam, 2002.

4 A.-C. Genix and J. Oberdisse, Current Opinion in Colloid \& Interface Science, 2015, 20, 293 303.

5 S. Kim, K. Hyun, B. Struth, K. H. Ahn and C. Clasen, Macromolecules, 2016, 49, 9068-9079.

6 N. Jouault, D. Lee, D. Zhao and S. K. Kumar, Advanced Materials, 2014, 26, 4031-4036.

7 A. Banc, A. C. Genix, M. Chirat, C. Dupas, S. Caillol, M. Sztucki and J. Oberdisse, Macromolecules, 2014, 47, 3219-3230.

8 J. Oberdisse and B. Deme, Macromolecules, 2002, 35, 4397-4405.

9 J. F. Berret, P. Herve, O. Aguerre-Chariol and J. Oberdisse, Journal of Physical Chemistry B, 2003, 107, 8111-8118.

10 J. Oberdisse, Y. Rharbi and F. Boue, Computational and Theoretical Polymer Science, 2000, 10, 207-217.

11 A.-C. Genix and J. Oberdisse, Soft Matter, 2017, 13, 8144-8155.

12 C. Schmitt Pauly, A.-C. Genix, J. G. Alauzun, J. Jestin, M. Sztucki, P. H. Mutin and J. Oberdisse, Polymer, 2016, 97, 138-146.

13 N. Jouault, F. Dalmas, F. Boue and J. Jestin, Polymer, 2012, 53, 761-775.

14 J. Oberdisse, P. Hine and W. Pyckhout-Hintzen, Soft Matter, 2007, 2, 476-485.

15 S. K. Kumar, N. Jouault, B. Benicewicz and T. Neely, Macromolecules, 2013, 46, 3199-3214.

16 G. P. Baeza, A. C. Genix, C. Degrandcourt, L. Petitjean, J. Gummel, M. Couty and J. Oberdisse, Macromolecules, 2013, 46, 317-329.

17 Y. Shinohara, H. Kishimoto, N. Yagi and Y. Amemiya, Macromolecules, 2010, 43, 9480-9487.

18 D. Stauffer and A. Aharony, Introduction To Percolation Theory., Taylor \& Francis, London, 1992.

19 H. Scher and R. Zallen, The Journal of chemical physics, 1970, 53, 3759-3761.

20 M. D. Rintoul and S. Torquato, Journal of Physics A: Mathematical and General, 1997, 30, L585-L592.

21 R. Ogata, T. Odagaki and K. Okazaki, Journal of Physics-condensed Matter, 2005, 17, 45314538 . 
22 U. Alon, A. Drory and I. Balberg, Phys. Rev. A, 1990, 42, 4634-4638.

23 E. T. Gawlinski and H. E. Stanley, Journal of Physics A: Mathematical and General, 1981, 14, L291-L299.

24 T. Vicsek and J. Kertesz, Journal of Physics A: Mathematical and General, 1981, 14, L31-L37.

25 M. J. Powell, Physical Review B, 1979, 20, 4194-4198.

26 R. Fantoni, D. Gazzillo and A. Giacometti, The Journal of chemical physics, 2005, 122, 034901.

27 Y. C. Chiew and E. D. Glandt, Journal of Physics A: Mathematical and General, 1983, 16, 25992608.

28 R. H. J. Otten and P. van der Schoot, Physical Review Letters, 2009, 103, 225704.

29 C. Grimaldi, The Journal of chemical physics, 2017, 147, 074502.

30 G. Ambrosetti, C. Grimaldi, I. Balberg, T. Maeder, A. Danani and P. Ryser, Phys. Rev. B, 2010, 81, 155434.

31 C. D. Lorenz and R. M. Ziff, The Journal of chemical physics, 2001, 114, 3659-3661.

32 A. Corsi and P. D. Gujrati, Phys. Rev. E, 2006, 74, 061123.

33 G. P. Baeza, J. Oberdisse, A. Alegria, M. Couty and A. C. Genix, Phys. Chem. Chem. Phys., 2015, 17, 1660-1666.

34 Y. Jin and R. A. Gerhardt, ACS Applied Materials \& Interfaces, 2014, 6, 22264-22271.

35 G. A. Campbell, M. E. Zak and M. D. Wetzel, Rheologica Acta, 2018, 57, 197-216.

36 A. Mujtaba, M. Keller, S. Ilisch, H. J. Radusch, M. Beiner, T. Thurn-Albrecht and K. Saalwächter, ACS Macro Letters, 2014, 3, 481-485.

37 J. S. Meth, S. G. Zane, C. Chi, J. D. Londono, B. A. Wood, P. Cotts, M. Keating, W. Guise and S. Weigand, Macromolecules, 2011, 44, 8301-8313.

38 K. W. Stockelhuber, A. S. Svistkov, A. G. Pelevin and G. Heinrich, Macromolecules, 2011, 44, 4366-4381.

39 D. N. Voylov, A. P. Holt, B. Doughty, V. Bocharova, H. M. Meyer, S. Cheng, H. Martin, M. Dadmun, A. Kisliuk and A. P. Sokolov, ACS Macro Letters, 2017, 6, 68-72.

40 Q. Zhang and L. A. Archer, Langmuir, 2002, 18, 10435-10442.

41 A.-C. Genix, C. Schmitt-Pauly, J. G. Alauzun, T. Bizien, P. H. Mutin and J. Oberdisse, Macromolecules, 2017, 50, 7721-7729.

42 C. Chevigny, F. Dalmas, E. Di Cola, D. Gigmes, D. Bertin, F. Boué and J. Jestin, Macromolecules, 2011, 44, 122-133.

43 C. Schmitt Pauly, A.-C. Genix, J. G. Alauzun, M. Sztucki, J. Oberdisse and P. H. Mutin, Phys. Chem. Chem. Phys., 2015, 17, 19173-19182.

44 E. J. W. Verwey and J. T. G. Overbeek, Theory of the stability of lyophobic colloids, Amsterdam: Elsevier., 1948.

45 A. C. Balazs, T. Emrick and T. P. Russell, Science, 2006, 314, 1107-1110. 
46 G. Heinrich, M. Kluppel and T. A. Vilgis, Current Opinion in Solid State \& Materials Science, 2002, 6, 195-203.

47 S. S. Sarkawi, W. Kaewsakul, K. Sahakaro, W. K. Dierkes and J. W. M. Noordermeer, J. Rubber Res., 2015, 18, 203-233.

48 K. Suematsu, M. Arimura, N. Uchiyama and S. Saita, ACS Applied Nano Materials, 2018, 1, 2430-2437.

49 Y.-C. Lin, H.-L. Chen, T. Hashimoto and S.-A. Chen, Macromolecules, 2016, 49, 7535-7550.

50 A.-C. Genix, V. Bocharova, A. Kisliuk, B. Carroll, S. Zhao, J. Oberdisse and A. P. Sokolov, ACS Applied Materials \& Interfaces, 2018, 10, 33601-33610.

51 D. Musino, A.-C. Genix, C. Fayolle, A. Papon, L. Guy, N. Meissner, R. Kozak, P. Weda, T. Bizien, T. Chaussée and J. Oberdisse, Macromolecules, 2017, 50, 5138-5145.

52 D. Musino, A. C. Genix, T. Chaussée, L. Guy, N. Meissner, R. Kozak, T. Bizien and J. Oberdisse, Langmuir, 2018, 34, 3010-3020.

53 R. L. McGreevy and L. Pusztai, Molecular Simulation, 1988, 1, 359-367.

54 R. L. McGreevy, Journal of Physics: Condensed Matter, 2001, 13, R877-R913.

55 G. Tóth, Journal of Molecular Liquids, 2006, 129, 108-114.

56 P. Cassagnau, Polymer, 2008, 49, 2183-2196.

57 T. Davris, M. R. B. Mermet-Guyennet, D. Bonn and A. V. Lyulin, Macromolecules, 2016, 49, 7077-7087.

58 Q. Chen, S. Gong, J. Moll, D. Zhao, S. K. Kumar and R. H. Colby, ACS Macro Letters, 2015, 4, 398-402.

59 H. M. Smallwood, J Appl Phys, 1944, 15, 758-766.

60 P. Meakin, Journal of Sol-Gel Science and Technology, 1999, 15, 97-117.

61 J. Sumfleth, S. T. Buschhorn and K. Schulte, Journal of Materials Science, 2011, 46, 659-669.

62 K. Rishi, G. Beaucage, V. Kuppa, A. Mulderig, V. Narayanan, A. McGlasson, M. Rackaitis and J. Ilavsky, Macromolecules, 2018, 51, 7893-7904. 